\title{
Trapped Torsional Vibrations in Elastic Plates
}

\author{
Min K. Kang and Rui Huang \\ Department of Aerospace Engineering and Engineering \\ Mechanics \\ University of Texas at Austin \\ Austin, Texas 78712 \\ Email: ruihuang@mail.utexas.edu (Huang)
}

Terence Knowles

Texzec, Inc.

Round Rock, Texas 78681

Email: tknowles@texas.net

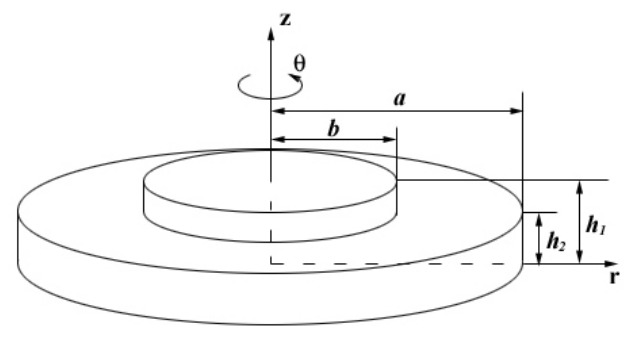

(a)

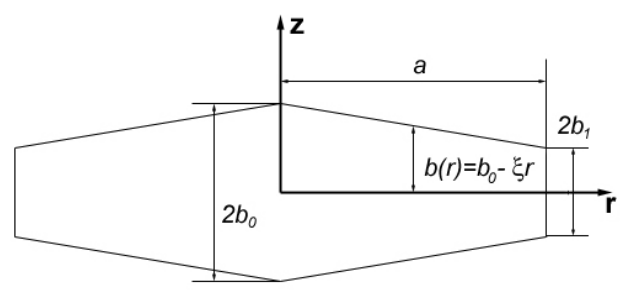

(b)

Fig. 1: (a) A circular plate with a thickness step; (b) An axisymmetrically contoured plate (cross-sectional view).

A well-known problem with the thickness-shear mode QCM is that in-plane shear motion intrinsically couples to out-of-plane flexure. When used in contact with liquids, the out-of-plane motion generates compressional waves that reflect off the liquid surface and return to the crystal. This interference effect causes depth-sensitive perturbations in the sensor responses [10,11]. Recently, we have discovered that torsional vibrations can be trapped in elastic plates with circular regions of slightly thicker steps or with smooth convex contoured surfaces [12]. In an elastically isotropic plate, torsional modes do not couple to flexural modes, have no out-of-plane motions, and hence no interference effect in contact with liquids, allowing an improved sensor response compared to thickness-shear-mode resonators. An electromagnetic acoustic transducer (EMAT) was used to generate oscillatory surface tractions that drive torsional vibrations of the plate. A similar method was previously used to generate trapped torsional waves in stepped cylinders [13].

This paper presents a theoretical study of torsional vibrations of isotropic elastic plates. First, a set of approximate equations is developed for vibrations of axisymmetrically contoured elastic plates, from which the governing equations for torsional modes are separated from

This research is funded in part by National Science Foundation through Grant CMS-0547409. 
flexural and extensional modes. Approximate solutions are then obtained for torsional vibrations of circular plates with thickness steps (Fig. 1a) and with linearly contoured faces (Fig. 1b). Energy trapping is predicted in an infinite plate with a stepped or contoured region, given that the geometrical parameters of the step or contour satisfy a critical condition. The theoretical results are compared to experiments and finite element analyses.

\section{Two-Dimensional Plate EQuations}

A general procedure for deducing approximate equations for elastic plates from the three-dimensional theory of linear elasticity was first introduced by Mindlin [14] based on the series expansion methods and the variational principle. The procedure has been used to derive approximate plate theories for both elastic and piezoelectric crystal plates with uniform [15-17] and nonuniform thickness [18-21]. Following the same procedure, we derive a set of approximate equations for axisymmetrically contoured elastic plates in this section, and develop analytical solutions for torsional vibrations in stepped and linearly contoured circular plates in Sections III and IV, respectively.

The Hamilton's principle of three-dimensional linear elasticity [22] can be written as:

$$
\int_{t_{0}}^{t} d t \int_{V}\left(\sigma_{i j, j}-\rho \ddot{u}_{i}\right) \delta u_{i} d V-\int_{t_{0}}^{t} d t \int_{S}\left(\sigma_{i j} n_{j}-t_{i}\right) \delta u_{i} d S=0,
$$

where $V$ is the volume of the solid bounded by the surface $S$, $\sigma_{i j}$ is the stress tensor, $t_{j}$ the surface traction, $u_{j}$ the displacement, $\rho$ the mass density, and $n_{j}$ the normal vector of the surface.

Consider an axisymmetrically contoured plate as shown in Fig. 1b. The top and bottom surfaces of the plate are symmetrically located at $z= \pm b(r)$, with the thickness $2 b(r)$ varying as a function of the radial coordinate measured from the center. Assuming a small surface gradient, the displacement can be expanded into a trigonometric series similar to that for a uniform plate $[15,16]$, namely

$$
u_{i}(r, \theta, z, t)=\sum_{n=0}^{\infty} u_{i}^{(n)}(r, \theta, t) \cos \left[\frac{n \pi}{2}(1-\psi)\right],
$$

where $\psi=z / b(r)$.

By substituting the series expansion (2) into the variational statement (1) and integrating over the thickness of the plate, we obtain a set of two-dimensional (2D) field equations and the corresponding boundary conditions. In cylindrical coordinates, the $n$ th-order $2 \mathrm{D}$ equations of motion are

$$
\begin{aligned}
& \sigma_{r r, r}^{(n)}+\frac{1}{r}\left(\sigma_{r \theta, \theta}^{(n)}+\sigma_{r r}^{(n)}-\sigma_{\theta \theta}^{(n)}\right)-\frac{n \pi}{2 b} \hat{\sigma}_{z r}^{(n)}+\frac{1-\delta_{n 0}}{2} \frac{b^{\prime}}{b} \sigma_{r r}^{(n)} \\
& +\frac{1}{b} F_{r}^{(n)}=\left(1+\delta_{n 0}\right) \rho \ddot{u}_{r}^{(n)} \\
& \sigma_{r \theta, r}^{(n)}+\frac{1}{r}\left(\sigma_{\theta \theta, \theta}^{(n)}+2 \sigma_{r \theta}^{(n)}\right)-\frac{n \pi}{2 b} \hat{\sigma}_{\theta z}^{(n)}+\frac{1-\delta_{n 0}}{2} \frac{b^{\prime}}{b} \sigma_{r \theta}^{(n)} \\
& \quad+\frac{1}{b} F_{\theta}^{(n)}=\left(1+\delta_{n 0}\right) \rho \ddot{u}_{\theta}^{(n)} \\
& \sigma_{r z, r}^{(n)}+\frac{1}{r}\left(\sigma_{z \theta, \theta}^{(n)}+\sigma_{r z}^{(n)}\right)-\frac{n \pi}{2 b} \hat{\sigma}_{z z}^{(n)}+\frac{1-\delta_{n 0}}{2} \frac{b^{\prime}}{b} \sigma_{r z}^{(n)} \\
& \quad+\frac{1}{b} F_{z}^{(n)}=\left(1+\delta_{n 0}\right) \rho \ddot{u}_{z}^{(n)}
\end{aligned}
$$

where $b^{\prime}=\frac{d b}{d r}$, and $\delta_{m n}=1$ if $m=n$ but 0 otherwise. Similar equations have been obtained previously for contoured crystal strips in rectangular coordinates [20]. The 2D components of the stress and the face traction are defined by

$$
\begin{aligned}
& \sigma_{i j}^{(n)}=\int_{-1}^{1} \sigma_{i j} \cos \frac{n \pi}{2}(1-\psi) d \psi \\
& \hat{\sigma}_{i j}^{(n)}=\int_{-1}^{1} \sigma_{i j} \sin \frac{n \pi}{2}(1-\psi) d \psi \\
& F_{j}^{(n)}=\sigma_{z j}(z=b)-(-1)^{n} \sigma_{z j}(z=-b) .
\end{aligned}
$$

The corresponding boundary conditions at the edge of the plate are:

$$
\begin{aligned}
& t_{r}^{(n)}=n_{r} \sigma_{r r}^{(n)}+n_{\theta} \sigma_{r \theta}^{(n)} \text { or } u_{r}^{(n)}=\hat{u}_{r}^{(n)}, \\
& t_{\theta}^{(n)}=n_{r} \sigma_{r \theta}^{(n)}+n_{\theta} \sigma_{\theta \theta}^{(n)} \text { or } u_{\theta}^{(n)}=\hat{u}_{\theta}^{(n)}, \\
& t_{z}^{(n)}=n_{r} \sigma_{r z}^{(n)}+n_{\theta} \sigma_{z \theta}^{(n)} \text { or } u_{z}^{(n)}=\hat{u}_{z}^{(n)},
\end{aligned}
$$

where $t_{i}^{(n)}=\int_{-1}^{1} t_{i} \cos \frac{n \pi}{2}(1-\psi) d \psi$, and $\hat{u}_{i}^{(n)}$ is prescribed edge displacements.

Associated with the series expansion of displacement in (2), the strain components can be obtained in form of similar expansions of 2D components. Then, applying Hooke's law of linear elasticity and integrating the stresses with respect to the plate thickness in (4), we obtain the 2D stress-strain relations. For the interest of space, only the first-order relations are given below.

For the first-order 2D equations, we apply a truncation procedure, assuming that

$$
\begin{aligned}
& u_{r}^{(n)}=u_{\theta}^{(n)}=0, \quad n>1 \\
& u_{z}^{(n)}=0, \quad n>2
\end{aligned}
$$

It is a common practice to retain a second-order term, $u_{z}^{(2)}$, in deducing the first-order equations. The extra term is eliminated from the equations later by setting $\sigma_{z z}^{(1)}=0$ and $\ddot{u}_{z}^{(2)}=0[14]$. 
In terms of stresses, the first-order 2D equations of motion are obtained from (3) with $n=0$ and 1 , for the zeroth and first-order modes, respectively. The first-order 2D stress-strain relations include:

$$
\begin{aligned}
& \sigma_{r r}^{(0)}=2(\lambda+2 \mu) u_{r, r}^{(0)}+2 \lambda\left(\frac{u_{r}^{(0)}}{r}+\frac{u_{\theta, \theta}^{(0)}}{r}\right)+\frac{2 \lambda}{b} u_{z}^{(1)} \\
& \sigma_{\theta \theta}^{(0)}=2 \lambda u_{r, r}^{(0)}+2(\lambda+2 \mu)\left(\frac{u_{r}^{(0)}}{r}+\frac{u_{\theta, \theta}^{(0)}}{r}\right)+\frac{2 \lambda}{b} u_{z}^{(1)} \\
& \sigma_{z z}^{(0)}=2 \lambda u_{r, r}^{(0)}+2 \lambda\left(\frac{u_{r}^{(0)}}{r}+\frac{u_{\theta, \theta}^{(0)}}{r}\right)+\frac{2}{b}(\lambda+2 \mu) u_{z}^{(1)} \\
& \sigma_{r \theta}^{(0)}=2 \mu\left(u_{\theta, r}^{(0)}-\frac{u_{\theta}^{(0)}}{r}+\frac{u_{r, \theta}^{(0)}}{r}\right) \\
& \sigma_{z \theta}^{(0)}=2 \mu \frac{u_{z, \theta}^{(0)}}{r}+\frac{2 \mu}{b} u_{\theta}^{(1)} \\
& \sigma_{z r}^{(0)}=2 \mu u_{z, r}^{(0)}+\frac{2 \mu}{b} u_{r}^{(1)}
\end{aligned}
$$

and

$$
\begin{aligned}
& \sigma_{r r}^{(1)}=\frac{4 \mu \lambda+4 \mu^{2}}{\lambda+2 \mu}\left(u_{r, r}^{(1)}-\frac{b^{\prime}}{2 b} u_{r}^{(1)}\right)+\frac{2 \mu \lambda}{\lambda+2 \mu}\left(\frac{u_{r}^{(1)}}{r}+\frac{u_{\theta, \theta}^{(1)}}{r}\right) \\
& \sigma_{\theta \theta}^{(1)}=\frac{2 \mu \lambda}{\lambda+2 \mu}\left(u_{r, r}^{(1)}-\frac{b^{\prime}}{2 b} u_{r}^{(1)}\right)+\frac{4 \mu \lambda+4 \mu^{2}}{\lambda+2 \mu}\left(\frac{u_{r}^{(1)}}{r}+\frac{u_{\theta, \theta}^{(1)}}{r}\right) \\
& \sigma_{r \theta}^{(1)}=\mu\left(u_{\theta, r}^{(1)}-\frac{u_{\theta}^{(1)}}{r}+\frac{u_{r, \theta}^{(1)}}{r}-\frac{b^{\prime}}{2 b} u_{\theta}^{(1)}\right) \\
& \sigma_{z \theta}^{(1)}=\mu \frac{u_{z, \theta}^{(1)}}{r} \\
& \sigma_{z r}^{(1)}=\mu u_{z, r}^{(1)}-\mu \frac{b^{\prime}}{2 b} u_{z}^{(1)}
\end{aligned}
$$

and

$$
\begin{aligned}
\hat{\sigma}_{z r}^{(1)} & =\mu\left(\frac{4}{\pi} u_{z, r}^{(0)}+\frac{\pi}{2 b} u_{r}^{(1)}\right) \\
& +\frac{\mu \lambda}{\pi(\lambda+2 \mu)}\left[\frac{b}{2}\left(u_{r, r r}^{(1)}+\frac{u_{r, r}^{(1)}}{r}-\frac{u_{r}^{(1)}}{r^{2}}+\frac{u_{\theta, \theta r}^{(1)}}{r}-\frac{u_{\theta, \theta}^{(1)}}{r^{2}}\right),\right. \\
& \left.-\frac{5}{6} b^{\prime}\left(\frac{13}{10} u_{r, r}^{(1)}+\frac{u_{r}^{(1)}}{r}+\frac{u_{\theta, \theta}^{(1)}}{r}\right)-\frac{b^{\prime \prime}}{4} u_{r}^{(1)}+\frac{2 b^{\prime 2}}{3 b} u_{r}^{(1)}\right] \\
\hat{\sigma}_{z \theta}^{(1)} & =\mu\left(\frac{4}{\pi} \frac{u_{z, \theta}^{(0)}}{r}+\frac{\pi}{2 b} u_{\theta}^{(1)}\right) \\
& +\frac{\mu \lambda}{2 \pi(\lambda+2 \mu)}\left(u_{r, r \theta}^{(1)}+\frac{u_{r, \theta}^{(1)}}{r}+\frac{u_{\theta, \theta \theta}^{(1)}}{r}-\frac{b^{\prime}}{2 b} u_{r, \theta}^{(1)}\right),
\end{aligned}
$$

$$
\hat{\sigma}_{z z}^{(1)}=\frac{4 \lambda}{\pi}\left(u_{r, r}^{(0)}+\frac{u_{r}^{(0)}}{r}+\frac{u_{\theta, \theta}^{(0)}}{r}\right)+(\lambda+2 \mu) \frac{\pi}{2 b} u_{z}^{(1)},
$$

where $\lambda$ and $\mu$ are the Lame constants of the elastic material.

Substituting (7), (8), and (9) into (3) leads to the firstorder 2D displacement equations of motion. By assuming circumferentially independent motion (i.e., $\partial / \partial \theta=0$ ), the equations can be separated into four uncoupled groups as follows.

First, the zeroth-order radial extension and the firstorder thickness-stretch are governed by

$$
\begin{aligned}
&(\lambda+2 \mu)\left(u_{r, r r}^{(0)}+\frac{u_{r, r}^{(0)}}{r}-\frac{u_{r}^{(0)}}{r^{2}}\right)+\frac{\lambda}{b} u_{z, r}^{(1)}-\frac{\lambda b^{\prime}}{b^{2}} u_{z}^{(1)}+\frac{1}{2 b} F_{r}^{(0)} \\
&=\rho \ddot{u}_{r}^{(0)} \\
& \mu\left(u_{z, r r}^{(1)}+\frac{u_{z, r}^{(1)}}{r}\right)-(\lambda+2 \mu) \frac{\pi^{2}}{4 b^{2}} u_{z}^{(1)}-\frac{\mu}{2}\left(\frac{b^{\prime \prime}}{b}+\frac{b^{\prime 2}}{2 b^{2}}-\frac{b^{\prime}}{b r}\right) u_{z}^{(1)} . \\
& \quad-\frac{2 \lambda}{b}\left(u_{r, r}^{(0)}+\frac{u_{r}^{(0)}}{r}\right)+\frac{1}{b} F_{z}^{(1)}=\rho \ddot{u}_{z}^{(1)}
\end{aligned}
$$

Second, for the flexure and the first-order thicknessshear modes, the equations of motion are

$$
\begin{aligned}
& \mu\left(u_{z, r r}^{(0)}+\frac{u_{z, r}^{(0)}}{r}\right)+\frac{\mu}{b}\left(u_{r, r}^{(1)}+\frac{u_{r}^{(1)}}{r}-\frac{b^{\prime}}{b} u_{r}^{(1)}\right)+\frac{1}{2 b} F_{z}^{(0)} \\
& =\rho \ddot{u}_{z}^{(0)} \\
& \frac{15 \mu \lambda+16 \mu^{2}}{4(\lambda+2 \mu)}\left(u_{r, r r}^{(1)}+\frac{u_{r, r}^{(1)}}{r}-\frac{u_{r}^{(1)}}{r^{2}}\right)-\mu \frac{\pi^{2}}{4 b^{2}} u_{r}^{(1)} \\
& -\mu\left[\frac{15 \lambda+16 \mu}{8(\lambda+2 \mu)} \frac{b^{\prime \prime}}{b}-\frac{16 \lambda+12 \mu}{12(\lambda+2 \mu)} \frac{b^{\prime 2}}{b^{2}}+\frac{5 \lambda+24 \mu}{12(\lambda+2 \mu)} \frac{b^{\prime}}{b} \frac{1}{r}\right] u_{r}^{(1)} \\
& -\frac{19 \mu \lambda}{24(\lambda+2 \mu)} \frac{b^{\prime}}{b} u_{r, r}^{(1)}-\frac{2 \mu}{b} u_{z, r}^{(0)}+\frac{1}{b} F_{r}^{(1)}=\rho \ddot{u}_{r}^{(1)}
\end{aligned}
$$

The two remaining equations are for the zeroth-order and the first-order circumferential motion, which are uncoupled from each other:

$$
\begin{aligned}
& \mu\left(u_{\theta, r r}^{(0)}+\frac{u_{\theta, r}^{(0)}}{r}-\frac{u_{\theta}^{(0)}}{r^{2}}\right)+\frac{1}{2 b} F_{\theta}^{(0)}=\rho \ddot{u}_{\theta}^{(0)} \\
& \mu\left(u_{\theta, r r}^{(1)}+\frac{u_{\theta, r}^{(1)}}{r}-\frac{u_{\theta}^{(1)}}{r^{2}}\right)-\frac{\mu}{2}\left(\frac{b^{\prime \prime}}{b}+\frac{b^{\prime 2}}{2 b^{2}}-\frac{3 b^{\prime}}{b r}-\frac{\pi^{2}}{2 b^{2}}\right) u_{\theta}^{(1)} \\
& +\frac{1}{b} F_{\theta}^{(1)}=\rho \ddot{u}_{\theta}^{(1)}
\end{aligned}
$$

The remainder of this paper focuses on the circumferential motion, corresponding to torsional modes in the elastic plates [23]. The uncoupling of torsional modes from flexural modes allows pure in-plane motion of the 
resonator, which is advantageous for applications requiring immersion in or in contact with liquids [12]. It is evident from Eq. (12) that the zeroth-order torsional motion is independent of contouring. This dictates that, under the assumption of small-gradient axisymmetric contouring, the zeroth-order torsional modes have the same solution as for a uniform plate. On the other hand, the first-order torsional motion in Eq. (13) depends on surface contouring, which makes it possible for energy trapping. Previous studies of straight-crested waves in rectangular plates have shown that two correction factors must be introduced to improve the lowest flexural and extensional branches of the dispersion curves $[15,16,20]$. For the torsional modes governed by Eqs. (12) and (13), however, no correction factor is needed.

\section{Torsional Vibrations of Stepped Plates}

For a stepped circular plate as shown in Fig. 1(a), we develop an approximate method to analyze the torsional vibrations by coupling two uniform plates of different thicknesses for the inner and outer regions through a mixed boundary condition at the junction [23]. By setting $b$ to be a constant (i.e., $b^{\prime}=b^{\prime \prime}=0$ ) in Eqs. (12) and (13) for a circular plate of uniform thickness, the $2 \mathrm{D}$ displacement equations of torsional motion can be written as

$$
\begin{aligned}
& \mu\left[\frac{\partial^{2} u_{\theta}^{(n)}}{\partial r^{2}}+\frac{1}{r} \frac{\partial u_{\theta}^{(n)}}{\partial r}-\frac{u_{\theta}^{(n)}}{r^{2}}\right]-\mu\left(\frac{n \pi}{h}\right)^{2} u_{\theta}^{(n)}+\frac{2-\delta_{n 0}}{h} F_{\theta}^{(n)}, \\
& =\rho \ddot{u}_{\theta}^{(n)}
\end{aligned}
$$

where $n=0,1$, and $h=2 b$ is the plate thickness. These 2D equations yield the exact solutions for zeroth- and first-order torsional modes in uniform plates without any correction factors [23].

Solving Eq. (14) for the inner region of the stepped plate $(r<b)$, we obtain that

$$
u_{\theta 1}^{(n)}=A_{1}^{(n)} J_{1}\left(\beta_{1}^{(n)} r\right) e^{i \omega t},
$$

where

$$
\beta_{1}^{(n)^{2}}=\frac{\rho}{\mu} \omega^{2}-\left(\frac{n \pi}{h_{1}}\right)^{2}
$$

For the outer region $(b<r<a)$, we have

$$
\begin{aligned}
& u_{\theta 2}^{(m)}=\left[A_{2}^{(m)} J_{1}\left(\beta_{2}^{(m)} r\right)+B_{2}^{(m)} Y_{1}\left(\beta_{2}^{(m)} r\right)\right] e^{i \omega t}, \\
& \beta_{2}^{(m)^{2}}=\frac{\rho}{\mu} \omega^{2}-\left(\frac{m \pi}{h_{2}}\right)^{2} .
\end{aligned}
$$

The corresponding shear stresses are

$$
\sigma_{r \theta 1}^{(n)}=-\frac{1+\delta_{n 0}}{2} \mu A_{1}^{(n)} \beta_{1}^{(n)} J_{2}\left(\beta_{1}^{(n)} r\right) e^{i \omega t},
$$

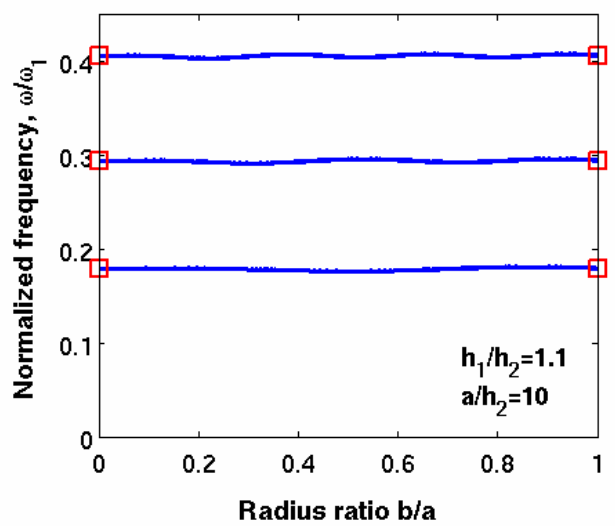

Fig. 2: Frequency spectrum of the zeroth-order trosional vibrations of circular plates with a thickness step $\left(h_{1} / h_{2}=1.1, a / h_{2}=10\right)$.

$$
\sigma_{r \theta 2}^{(m)}=-\frac{1+\delta_{m 0}}{2} \mu \beta_{2}^{(m)}\left[A_{2}^{(m)} J_{2}\left(\beta_{2}^{(m)} r\right)+B_{2}^{(m)} Y_{2}\left(\beta_{2}^{(m)} r\right)\right] e^{i \omega t} .
$$

For $h_{1}>h_{2}$, both the displacement and the traction are required to be continuous at the junction $(r=b)$ for $0<z<h_{2}$. In addition, part of the edge of the inner plate $\left(h_{2}<z<h_{1}\right)$ is traction free with unspecified displacement. Based on the series expansion of the displacement and the integral definition of the 2D tractions, an approximation of the mixed boundary condition is given by [23]

$$
\begin{gathered}
u_{\theta 2}^{(m)}=\frac{2-\delta_{m 0}}{h_{2}} \int_{0}^{h_{2}} u_{\theta 1}^{(n)} \cos \left(n \pi \frac{z}{h_{1}}\right) \cos \left(m \pi \frac{z}{h_{2}}\right) d z, \\
\sigma_{r \theta 1}^{(n)}=\frac{1}{h_{1}} \int_{0}^{h_{2}} \sum_{m=0}^{\infty}\left(2-\delta_{m 0}\right) \sigma_{r \theta 2}^{(m)} \cos \left(m \pi \frac{z}{h_{2}}\right) \cos \left(n \pi \frac{z}{h_{1}}\right) d z .
\end{gathered}
$$

In addition, a traction-free condition at the outer edge $(r=a)$ requires that

$$
J_{2}\left(\beta_{2}^{(m)} a\right) A_{2}^{(m)}+Y_{2}\left(\beta_{2}^{(m)} a\right) B_{2}^{(m)}=0
$$

\section{A. Zeroth-order torsional modes}

For the zeroth-order modes, we take $m=n=0$ for the inner and outer regions of the stepped plate. The resonance frequencies and mode shapes are determined from Eqs. (21) and (22). Figure 2 shows the first three resonance frequencies of the zeroth-order modes varying with the radius ratio $b / a$ for a fixed thickness ratio, $h_{1} / h_{2}=1.1$. The normalized outer radius is $a / h_{2}=10$. The frequencies are normalized by the first cut-off frequency of the inner plate, $\omega_{1}=\frac{\pi}{h_{1}} \sqrt{\frac{\mu}{\rho}}$. At both ends of the plot, with the radius ratio $b / a$ being 0 or 1 , the stepped plate recovers uniform circular plates with thickness $h_{2}$ and $h_{1}$, respectively. The resonance 
frequencies of the zeroth-order modes in a uniform circular plate are labeled in Fig. 2 as open squares. It is interesting to note that, while the resonance frequencies of zeroth-order torsional modes are independent of the thickness for a uniform circular plate, the frequencies in the stepped plate oscillate slightly as the radius ratio $b / a$ varies, possibly due to scattering at the stepped boundary.

\section{B. First-order torsional modes}

For the first-order modes, we take $n=1$ for the inner region and $m=0$ and 1 for the outer region. Figure 3 shows the frequency spectrum for the first-order torsional vibrations with $h_{1} / h_{2}=1.1$ and $a / h_{2}=10$. The left end of the plot corresponds to a uniform circular plate of thickness $h_{2}$, which has a higher cut-off frequency, $\omega_{2}=\frac{\pi}{h_{2}} \sqrt{\frac{\mu}{\rho}}$, compared to the cut-off frequency $\omega_{1}$ for a uniform plate of thickness $h_{1}$ at the right end. In between, the resonance frequencies change continuously. No first-order modes can be found below the cut-off frequency $\omega_{1}$. Between the two cut-off frequencies, the first-order modes in the inner plate are coupled with the zeroth-order modes in the outer plate, while the first-order modes in the outer plate are non-propagating.

\section{TORSIONAL Vibrations OF LinEARLy CONTOURED PLATES}

Consider a linearly contoured circular plate (Fig. 1b) with $b(r)=b_{0}-\xi r$, where $b_{0}$ is the half-thickness at the center and $\xi$ is the slope of the contour from the center to the edge ( $\xi<<1$ by the small gradient assumption). For free vibrations in the first-order torsional modes, by setting $F_{\theta}^{(1)}=0$ and $u_{\theta}^{(1)}=u(r) e^{i \omega t}$ in Eq. (13), we obtain that

$$
\begin{gathered}
u^{\prime \prime}+\frac{1}{r} u^{\prime}-\frac{1}{r^{2}}\left[1-\frac{3 \xi r}{2\left(b_{0}-\xi r\right)}-\frac{\xi^{2} r^{2}}{4\left(b_{0}-\xi r\right)^{2}}\right. \\
\left.+\frac{\pi^{2}}{4}\left(\frac{r}{b_{0}}\right)^{2}\left(\frac{b_{0}^{2}}{\left(b_{0}-\xi r\right)^{2}}-\frac{\omega^{2}}{\omega_{0}^{2}}\right)\right] u=0
\end{gathered},
$$

where $\omega_{0}=\frac{\pi}{2 b_{0}} \sqrt{\frac{\mu}{\rho}}$ is the cut-off frequency for the firstorder torsional mode in a uniform plate of thickness $2 b_{0}$. For a uniform plate ( $\xi=0$ ), Eq. (23) reduces to a Bessel equation with solutions in the form of Bessel functions. For a contoured plate, Eq. (23) can be solved by the Frobenius method [20,21].

Let

$$
u(r)=\sum_{m=0}^{\infty} C_{m}\left(\frac{r}{b_{0}}\right)^{m+\alpha}
$$

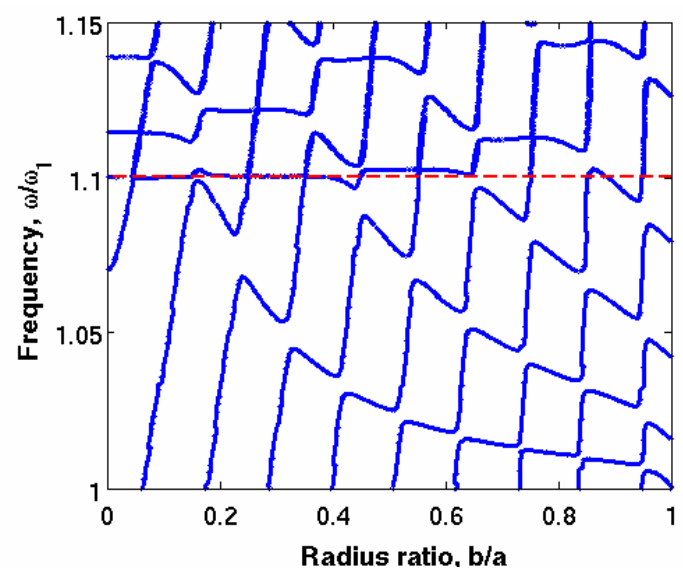

Figure 3: Frequency spectrum of the first-order torsional vibrations of circular plates with a thickness step $\left(h_{1} / h_{2}=1.1, a / h_{2}=10\right)$.

Substituting (24) into (23), we obtain that

$$
\begin{aligned}
& \sum_{m=0}^{\infty}\left[(m+\alpha)^{2}-1\right] C_{m} \bar{r}^{m+\alpha}-2 \xi \sum_{m=1}^{\infty}\left[(m+\alpha-1)^{2}-\frac{7}{4}\right] C_{m-1} \bar{r}^{m+\alpha} \\
& +\sum_{m=2}^{\infty}\left[\xi^{2}(m+\alpha-2)^{2}-\frac{9}{4} \xi^{2}+\frac{\pi^{2}}{4}\left(1-\Omega^{2}\right)\right] C_{m-2} \bar{r}^{m+\alpha} \\
& -\frac{\pi^{2}}{2} \xi \Omega^{2} \sum_{m=3}^{\infty} C_{m-3} \bar{r}^{m+\alpha}+\frac{\pi^{2}}{4} \xi^{2} \Omega^{2} \sum_{m=4}^{\infty} C_{m-4} \bar{r}^{m+\alpha}=0
\end{aligned}
$$

where $\bar{r}=r / b_{0}$ and $\Omega=\omega / \omega_{0}$. The left hand side of (25) is a polynomial function of $\bar{r}$. First, for the lowest power of the polynomial (i.e., $m=0$ ), we have

$$
\left(\alpha^{2}-1\right) C_{0}=0 \text {. }
$$

For a non-trivial solution, $C_{0}$ must not be zero, which leads to $\alpha= \pm 1$. Furthermore, the displacement must be finite at the center of the plate $(r=0)$, which requires that $\alpha=1$.

By setting all the coefficients of the polynomial at the left hand side of (25) to zero, we obtain the recurrence relations for $C_{m}$ :

$$
\begin{aligned}
& C_{1}=-\frac{1}{2} \xi C_{0}, \\
& C_{2}=\frac{9}{16} \xi C_{1}+\frac{5 \xi^{2}+\pi^{2}\left(1-\Omega^{2}\right)}{32} C_{0}, \\
& C_{3}=\frac{29}{30} \xi C_{2}-\frac{7 \xi^{2}-\pi^{2}\left(1-\Omega^{2}\right)}{60} C_{1}+\frac{\pi^{2} \Omega^{2}}{30} \xi C_{0},
\end{aligned}
$$

and for $m>3$

$$
\begin{aligned}
& C_{m}=\frac{4 m^{2}-7}{2 m(m+2)} \xi C_{m-1}-\left[\frac{4(m-1)^{2}-9}{4 m(m+2)} \xi^{2}-\frac{\pi^{2}\left(1-\Omega^{2}\right)}{4 m(m+2)}\right] C_{m-2} \\
& +\frac{\pi^{2} \Omega^{2}}{2 m(m+2)} \xi C_{m-3}-\frac{\pi^{2} \Omega^{2}}{4 m(m+2)} \xi^{2} C_{m-4}
\end{aligned}
$$




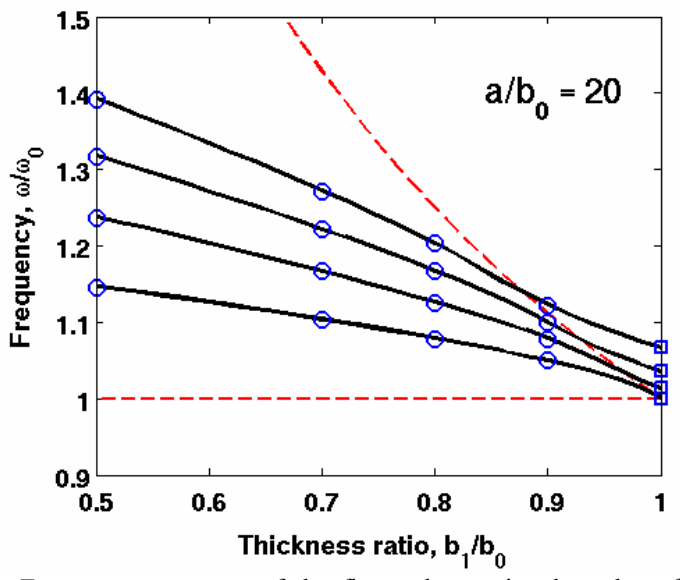

Fig. 4: Frequency spectrum of the first-order torsional modes of linearly contoured elastic plates $\left(\eta=a / b_{0}=20\right)$.

Applying the traction-free boundary condition at the edge of the plate, i.e., $\sigma_{r \theta}^{(1)}(r=a)=0$, we obtain that

$$
\sum_{m=1}^{\infty}\left(m C_{m}+\frac{\xi}{2(1-\xi \eta)} C_{m-1}\right) \eta^{m}=f(\Omega, \xi, \eta) C_{0}=0
$$

where $\eta=a / b_{0}$. Therefore, for given geometric parameters $\xi$ and $\eta$, the resonance frequencies of the contoured plate can be determined by setting $f(\Omega, \xi, \eta)=0$. It can be shown that, when $\xi=0$, the frequency equation reduces to that for uniform circular plates [23].

Figure 4 plots the resonant frequencies versus the thickness ratio, $b_{1} / b_{0}=1-\xi \eta$, for the first four torsional modes in contoured plates with $\eta=20$. The two dashed lines are plotted to denote the cut-off frequencies for the fundamental thickness mode in uniform plates of thickness $2 b_{0}$ (the center thickness) and $2 b_{1}$ (the edge thickness), respectively. For comparison, the exact solutions for uniform plates with $b_{1} / b_{0}=1$ are plotted as open squares, and results from finite element analyses (FEA) for $b_{1} / b_{0}=$ $0.9,0.8,0.7$, and 0.5 are plotted as open circles.

\section{ENERGY TRAPPING}

Energy trapping is critical for high-Q resonators. In thickness-shear mode quartz crystal resonators, partial electrodes are used to achieve energy trapping, for which the size of the electrodes has an upper limit given by Bechmann's number in order to eliminate anharmonic overtones [4-9]. The effectiveness of energy trapping can be further improved by using contoured crystal plates with decreasing thickness from the center to the edges, such as beveled and convex plates [18-21]. It was demonstrated that torsional waves can be trapped in stepped elastic cylinders [13], in which case the torsional waves are propagating in the axial direction in the region with a greater diameter but nonpropagating (evanescent) in the regions with a smaller diameter. Similarly, in a circular plate (with a diameter much

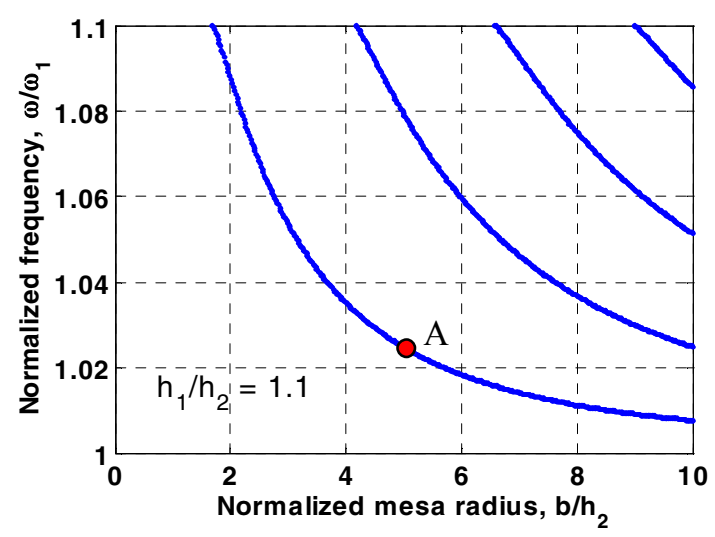

Fig. 5: The resonant frequencies of energy-trapped first-order torsional modes in a circular stepped infinite plate $\left(h_{1} / h_{2}=1.1, a \rightarrow \infty\right)$.
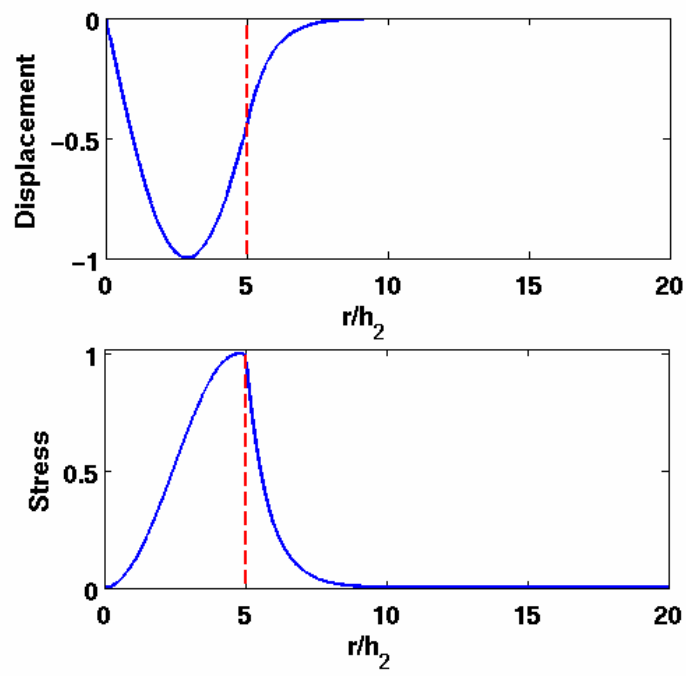

Fig. 6: Mode shapes of the first-order torsional mode corresponding to point A of Fig. $5\left(h_{1} / h_{2}=1.1, b / h_{2}=5\right)$, showing the characteristic of energy trapping.

greater than the thickness), trapped torsional modes can be achieved by varying the plate thickness, such as stepped or contoured plates (Fig. 1).

For a stepped plate as shown in Fig. 1a, between the two cut-off frequencies $\left(\omega_{1}<\omega<\omega_{2}\right)$, the first-order torsional modes are propagating in the inner region but nonpropagating in the outer region, implying energy trapping for the first-order modes. However, the situation is complicated by the coupling between the first-order modes of the inner plate and the zeroth-order modes of the outer plate (both propagating). Only when the coupling is weak and the firstorder mode dominates would energy trapping be effectively achieved. Theoretically, the zeroth-order modes are suppressed in an infinite plate (i.e., $a \rightarrow \infty$ ). In such cases, the solution to the torsional modes in the outer plate can be described by the first Hankel function that has an asymptote decaying exponentially away from the stepped region. Consequently, for any possible modes, energy is trapped 
within the stepped region. Figure 5 plots the resonance frequencies of the first-order torsional modes for thickness ratio, $h_{1} / h_{2}=1.1$. No trapped mode can be found when the radius of the stepped region $b / h_{2}<1.7$. For $1.7<b / h_{2}<4.2$, only one trapped torsional mode exists, with the resonance frequency decreasing as the radiusthickness ratio increases. For $b / h_{2}>4.2$, more than one trapped modes exist. Therefore, in addition to the upper limit for the step radius that is similar to the Bechmann's number for thickness-shear mode quartz crystal resonators, there is a lower limit for the step radius in order to have just one trapped torsional mode. Both the upper and the lower limits depend on the thickness ratio, $h_{1} / h_{2}$, as given in [23]. Figure 6 plots the mode shape corresponding to Point A in Fig. 5, showing the characteristic of trapped torsional modes. The displacement has a maximum at the inner region, and the maximum of the shear stress is near the edge of the stepped region. Both the displacement and the stress decay rapidly outside the stepped region. The mode shape also indicates that a finite plate of large outer radius (e.g., $a / h_{2}>20$ ) can be reasonably modeled as an infinite plate.

For a linearly contoured plate as shown in Fig. 1b, the coupling between first-order and zeroth-order modes are much weaker due to the smooth variation of thickness. Consequently, energy trapping can be effectively achieved even in a finite plate. Figure 7 shows the displacement mode shapes for linearly contoured plates with different thickness ratios, $b_{1} / b_{0}$, for $a / b_{0}=20$, corresponding to the lowest branch in Fig. 4. When $b_{1} / b_{0}=1$, the solution recovers that for a uniform plate, and the displacement is linear from the center to the edge. As the thickness ratio decreases, the location of the peak displacement moves toward the center. The mode shape shows the character of energy trapping when $b_{1} / b_{0}<0.9$. For comparison, FEA results are plotted as open circles.

To further illustrate the concept of energy trapping, we consider an infinite plate with an axisymmetrically contoured region (Fig. 8). The plate thickness varies linearly in the contoured region but remains constant $\left(b_{1}=b_{0}-\xi_{a}\right)$ elsewhere. For this case, the solution can be developed by combining the previous solution for contoured circular plates and that for a uniform annular plate through the continuity condition at the junction. For the contoured region $(r<a)$, the displacement takes the same form as (24), and the recurrence relations (27-30) remain valid. For the outer plate $(r>a)$, the displacement for the first-order torsional motion is,

$$
u(r)=D H_{1}^{(1)}(\beta r),
$$

where $D$ is a constant to be determined, $H_{1}^{(1)}$ denotes the first-order Hankel function of the first kind, and

$$
\beta^{2}=\frac{\rho}{\mu} \omega^{2}-\left(\frac{\pi}{2 b_{1}}\right)^{2} .
$$

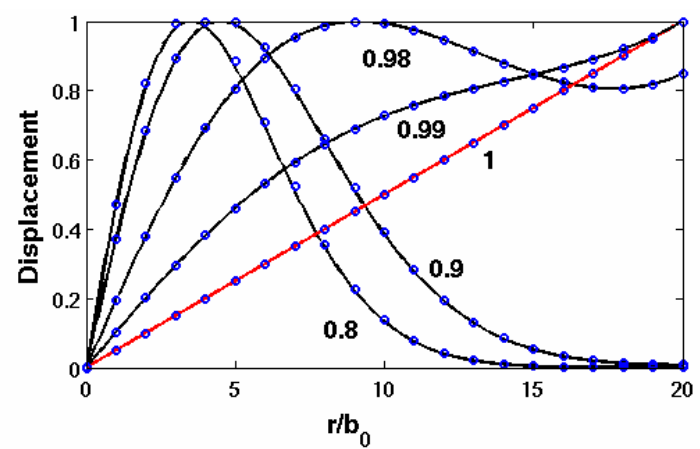

Fig. 7: Displacement mode shapes for linearly contoured plates with different thickness ratios with $\eta=a / b_{0}=20$.

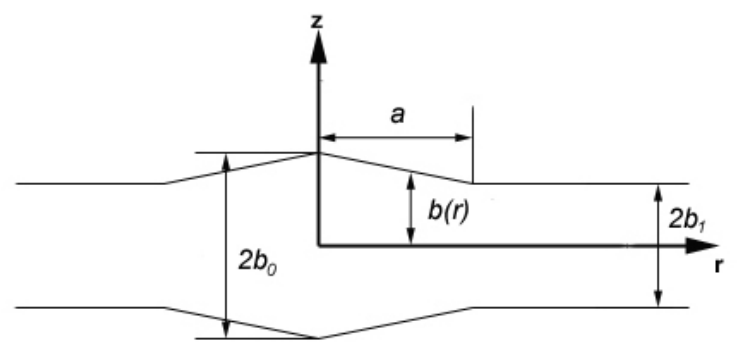

Fig. 8: Schematics of an infinite plate with an axisymmetrically contoured region for energy trapping.

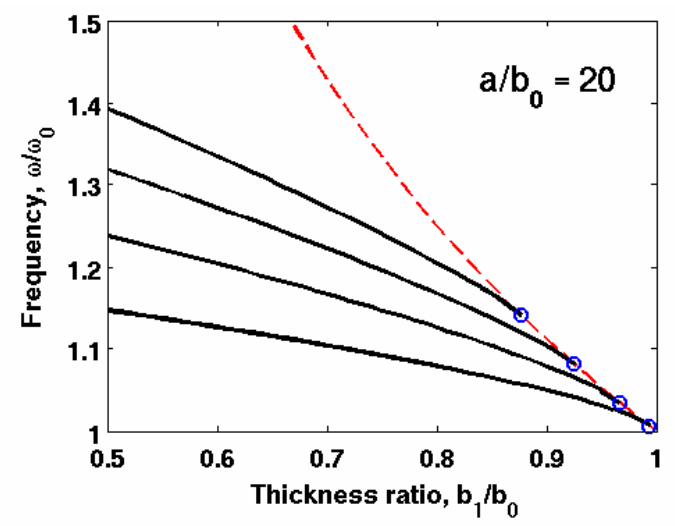

Fig. 9: Frequency spectrum of trapped first-order torsional modes in infinite plates with linearly contoured region.

At the junction $(r=a)$, both the displacement and the shear traction are continuous, which requires that

$$
\begin{gathered}
\sum_{m=0}^{\infty} C_{m} \eta^{m+1}=D H_{1}^{(1)}(\beta a), \\
\sum_{m=1}^{\infty}\left(m C_{m}-\frac{\xi}{2(1+\xi \eta)} C_{m-1}\right) \eta^{m}=-\beta D H_{2}^{(1)}(\beta a) .
\end{gathered}
$$

By applying the recurrence relations in (27)-(30), Eqs. (34) and (35) form a linear system with two constants, $C_{0}$ and $D$. For non-trivial solutions, the determinant of the coefficient matrix vanishes, which leads to a frequency equation for free vibrations of the infinite plate. Figure 9 plots the frequency spectrum for the first four branches. 
Now that the plate is infinite in the outer region, only trapped modes exist, and each branch of the frequency spectrum is bounded by the cut-off frequency in the outer region. For a fixed radius-thickness ratio, $a / b_{0}$, there exists an upper limit for the thickness ratio $b_{1} / b_{0}$, below which at least one trapped torsional mode exist.

The theoretical results from this study were compared to experimental measurements [12,23]. Circular steps and contoured regions were machined in a 6000 series cast aluminum plate. A non-contact electromagnetic acoustic transducer or EMAT was employed to generate oscillatory surface tractions. Torsional modes were excited when the traction force was applied in the circumferential direction. Resonant frequencies were determined by adjusting the pulse train frequency for peak initial amplitude as observed with an oscilloscope. A series of tests were conducted to measure the surface motion at resonances in order to confirm the observation of trapped torsional modes, for example, by using an absorbing stylus to determine the locations of displacement maxima and using a pick-up coil to confirm the circumferential motion. The agreement between the measured resonance frequencies and the theoretical results was excellent.

Finite element analysis (FEA) was also conducted to compare with the analytical solutions. A typical FEA eigenvalue problem gives many modes that are not of interest. Based on the approximate analytical solutions developed in this paper, a relatively narrow frequency window can be determined to reduce the number of eigen modes, from which the torsional modes can be identified by examining the mode shapes. Commercial FEA packages, ANSYS and ABAQUS, were used, and the results from both compare closely with the analytical solutions, as shown in Figs. 4 and 7.

\section{SUMMARY}

In this paper, torsional vibrations of circular plates with stepped and contoured thickness are analyzed by using approximate plate equations. For stepped plates, an approximate method is developed to couple the torsional modes in the uniform regions by a mixed boundary condition at the junction. For axisymmetrically contoured plates with a linear variation in thickness, the Frobenius method is used to solve the equation of motion. Of practical interest is energy trapping of the first-order torsional modes. Both stepped and contoured regions can be used to achieve energy trapping of torsional modes if a condition for geometrical parameters is satisfied. The analytical results are compared to experiments and FEA with good agreements.

\section{REFERENCES}

[1] C. Behling, R. Lucklum, P. Hauptmann, "Response of quartz-crystal resonators to gas and liquid analyte exposure." Sensors and Actuators A $68,388-398,1998$.
[2] J. Wegener, A. Janshoff, H.J. Galla, "Cell adhesion monitoring using a quartz crystal microbalance: comparative analysis of different mammalian cell lines.” Eur. Biophys. J. 28, 26-37, 1999.

[3] M. Kaspar, H. Stadler, T. Weib, C. Ziegler, "Thickness shear mode resonators ('mass-sensitive devices') in bioanalysis." Fresenius' J. Anal. Chem. 366, 602-610, 2000.

[4] R. Bechmann, "Quartz AT-type filter crystals for the frequency range 0.7 to 60 Mc.”, in Proc. Inst. Radio Eng, vol. 49, pp. 523-524, 1961.

[5] W. Shockley, D.R. Curran, D.J. Koneval, "Trapped-energy modes in quartz filter crystals," J. Acoust. Soc. Am. 41, 981-993, 1967.

[6] R.D. Mindlin, P.C.Y. Lee, "Thickness-shear and flexural vibrations of partially plated, crystal plates", Int. J. Solids Struct., vol. 2, pp. $125-139,1966$

[7] R.D. Mindlin, "Bechmann's number for harmonic overtones of thickness/twist vibrations of rotated Y-cut quartz plates." J. Acoust. Soc. Am. 41, 969-973, 1967.

[8] D.W. Haines, "Bechmann's number for harmonic overtones of thickness-shear vibrations in rotated Y-cut quartz plates." Int. J. Solids Struct. 5, 1-15, 1969.

[9] J.L. Bleustein, H.F. Tiersten, "Forced thickness-shear vibrations of discontinuously plated piezoelectric plates." J. Acoust. Soc. Am. 43, 1311-1318, 1968.

[10] T.W. Schneider, S.J. Martin, "Influence of compressional wave generation on thickness-shear mode resonator response in a fluid", Anal. Chem., vol. 67, pp. 3324-3335, 1995.

[11] P.C.Y. Lee, R. Huang, "Effects of a liquid layer on thickness-shear vibrations of rectangular AT-cut quartz plates", IEEE Trans. Ultrasonics, Ferroelectrics and Frequency Control, vol. 49, pp. 604$611,2002$.

[12] T. Knowles, M.K. Kang, R. Huang, "Trapped torsional vibrations in elastic plates", Appl. Phys. Lett., vol. 87, 201911, 2005.

[13] W. Johnson, B.A. Auld, E. Segal, F. Passarelli, "Trapped torsional modes in solid cylinders", J. Acoust. Soc. Am., vol. 100, pp. 285-293, 1996.

[14] R.D. Mindlin, "An introduction to the mathematical theory of vibrations of elastic plates", A monograph prepared for US Army Signal Corps Engineering Laboratories, Fort Monmouth, New Jersey, 1955.

[15] P.C.Y. Lee and Z. Nikodem, "An approximate theory for highfrequency vibrations of elastic plates." Int. J. Solids Structures 8, 581$612,1972$.

[16] P.C.Y. Lee, S. Syngellakis, J.P. Hou, "A two-dimensional theory for high-frequency vibrations of piezoelectric crystal plates with or without electrodes.” J. Appl. Phys., vol. 61, pp. 1249-1262, 1987.

[17] P.C.Y. Lee, J.D. Yu, and W.S. Lin, "A New Two-Dimensional Theory for Vibrations of Piezoelectric Crystal Plates with Electroded Faces.” J. Appl. Phys, 83, pp. 1213-1223, 1998.

[18] R.D. Mindlin, M. Forray, "Thickness-shear and flexural vibrations of contoured crystal plates”, J. Appl. Phys., vol. 25, pp. 12-20, 1954.

[19] D.S. Stevens and H.F. Tiersten, "An analysis of doubly rotated quartz resonators utilizing essentially thickness modes with transverse variation", J. Acoust. Soc. Am. 79, 1811-1826, 1986.

[20] P.C.Y. Lee, J. Wang, "Piezoelectrically forced thickness-shear and flexural vibrations of contoured quartz resonators", J. Appl. Phys., vol. 79, pp. 3411-3422, 1996.

[21] J. Wang, P.C.Y. Lee, D.H. Bailey, "Thickness-shear and flexural vibrations of linearly contoured crystal strips with multiprecision computation", Computers and Structures, vol. 70, pp. 437-445, 1999.

[22] K.F. Graff, Wave Motion In Elastic Solids. Dover Publications, Inc., New York, 1991, pp. 598-600.

[23] M.K. Kang, R. Huang, T. Knowles, "Torsional vibrations of circular elastic plates with thickness steps", IEEE Trans. Ultrasonics, Ferroelectrics, and Frequency Control, vol. 53, pp. 349-359, 2006. 